\title{
Widely tunable gain-switched operation of external cavity grating-coupled surface emitting laser
}

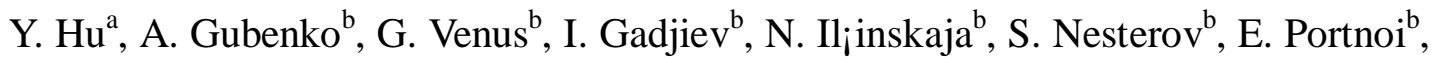 \\ M. Dubov ${ }^{a}$, I. Khrushchev ${ }^{\mathrm{a}}$ \\ a Photonics Research Group, Aston University, Aston Triangle, Birmingham B4 7ET, UK; \\ ${ }^{\mathrm{b}}$ Ioffe Institute, Russian Academy of Sciences, St. Petersburg 194021, Russia
}

\begin{abstract}
Widely tunable gain switching of a grating-coupled surface-emitting laser (GCSEL) has been demonstrated in a simple external cavity configuration for the first time. Pulse duration in range of 40-100ps and wavelength tuning over $100 \mathrm{~nm}$ have been achieved. High power, tail-free optical pulses have been observed at $980 \mathrm{~nm}$.
\end{abstract}

Keywords: wavelength tunable, gain switching, GCSEL, external feedback, ultrashort pulse, high power

\section{INTRODUCTION}

Recently, the grating-coupled surface emitting lasers (GCSELs) have become a subject of considerable research interest $^{1,2}$. Due to the built-in off-resonance grating structure, this type of laser offers the advantage of exceptionally good suppression of parasitic feedback as well as a possibility to employ the grating for the broadband spectral control. The GCSELs are therefore extremely well suited for the external cavity configurations. Indeed, the broadband, continuous tuning in $\mathrm{CW}$ regime over a range of $115 \mathrm{~nm}$ has been demonstrated ${ }^{1}$. One particularly interesting prospect is to use the GCSELs for building compact, tunable sources of ultrashort optical pulses. A preliminary study has shown that the GCSELs can be easily gain-switched and tuned over $60 \mathrm{~nm}$ spectral span to produce relatively long, $160 \mathrm{ps}^{\text {pulses }}{ }^{2}$. In this paper, we present the results of a more detailed study of the optimised operation of a GCSEL in the gain-switched regime, resulting in the tuning range of $100 \mathrm{~nm}$ with the pulsewidth in range of 40-100ps.

\section{EXPERIMENT}

The laser used in our experiment is a single quantum well graded-index (SQW GRIN) structure, composed of a $100 \mu \mathrm{m}$ wide, $500 \mu \mathrm{m}$ long active region with electrical confinement provided by $\mathrm{SiO}_{2}$ stripes. The active layer is followed by an $850 \mu \mathrm{m}$ long off-resonance grating section. Bragg resonance is not achieved in the grating for any frequencies inside the gain band, therefore no signal is reflected back into the laser from the grating. Instead, the light in the grating is coupled 
out of the laser under a certain angle to the surface (Fig.1).

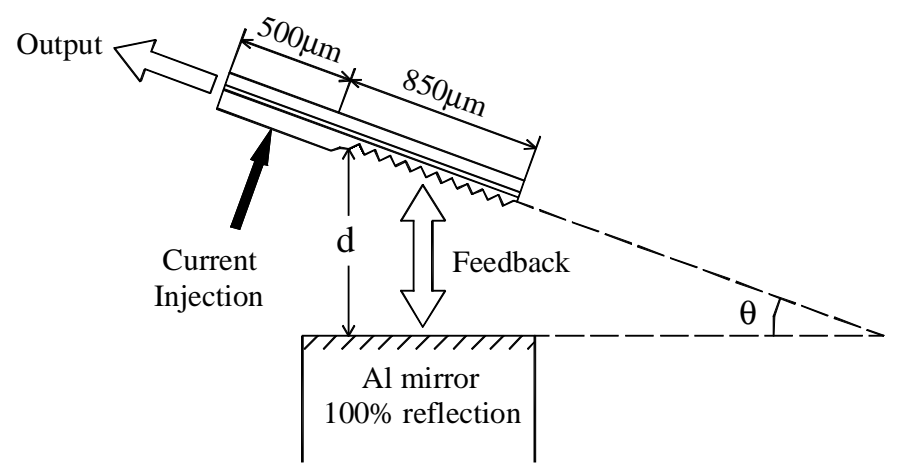

Fig.1: External cavity configuration

In the experiment, the external cavity was formed by a small ( $1 \mathrm{~mm}$ by $1 \mathrm{~mm}$ approximately) $100 \%$ reflecting aluminium flat mirror, placed in the vicinity of the grating section, as shown in the Fig.1. It was possible to vary the distance $d$ and the angle $\theta$ between the surfaces of mirror and the grating. The output was taken from the cleaved facet on the edge of the active region.

The laser was driven by an avalanche pulse generator with a repetition rate of $10 \mathrm{KHz}$ and the maximum current of 5A, with no DC bias. The electrical pulses had a rise-time of 150ps and a fall-time of 650ps.

\section{RESULTS AND DISCUSSION}

Lasing in the device only occurred when the external cavity was properly aligned. The output power increased as the mirror was moved closer to the laser. The signal wavelength was tuned by adjustment of the mirror angle $\theta$, but was not affected by the distance to the mirror $d$. We therefore kept the length of the external cavity very short, $800 \mu \mathrm{m}$ approximately, in order to provide strong optical feedback.

When the mirror angle was adjusted from $15.9^{\circ}$ to $35.0^{\circ}$, the generation wavelength changed by $100 \mathrm{~nm}$ from $902 \mathrm{~nm}$ to $1002 \mathrm{~nm}$. The tuning curves, showing the peak power and the pulsewidth as functions of wavelength, are shown in Fig.2.

The pulse duration increased towards the edges of the tuning curve. The pulse duration shorter than $100 \mathrm{ps}$ was observed within the 70nm-wide spectral window (from 920-990nm). The shortest pulse duration of 41ps was obtained at a wavelength of $938 \mathrm{~nm}$ with the corresponding peak power of $0.64 \mathrm{~W}$. The maximum peak power of $0.88 \mathrm{~W}$ was observed at a wavelength of $980 \mathrm{~nm}$. The pulse was tail-free and 57ps long at this point (Fig.3). 


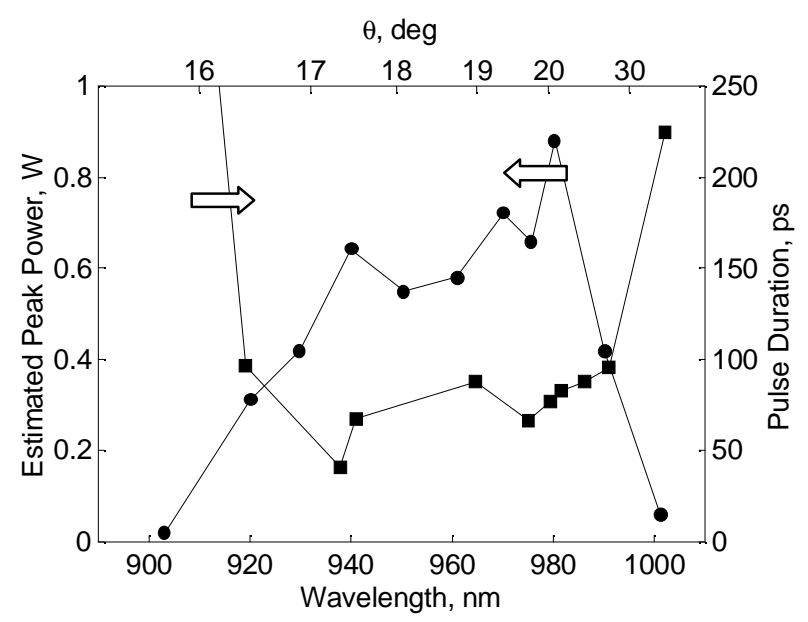

Fig.2: Tuning curves of the laser. Peak power and the pulse duration are shown over the entire tuning range.

We found that the spectra changed periodically between single mode and multimode when tuning the angle $\theta$. The wavelength interval between adjacent single mode states was $1.18 \mathrm{~nm}$. At the edge of the whole wavelength tuning range, multimode operation disappeared; instead, dark points between neighbouring single modes were found. The phenomenon was likely to be caused by the etalon effect in the grating substrate. The period observed approximately matched the thickness and refractive index of the substrate. The greatest peak power was achieved at a wavelength within the single mode range, with the FWHM spectral bandwidth of $0.18 \mathrm{~nm}$. The duration-bandwidth product at this point is in range of 2.2-3, indicating the possibility to compress the pulses by approximately a factor of 6 in a dispersion delay line. Exceptionally good spectral side-band suppression ratio of 40dB was observed in single-mode regime (inset of Fig.3).

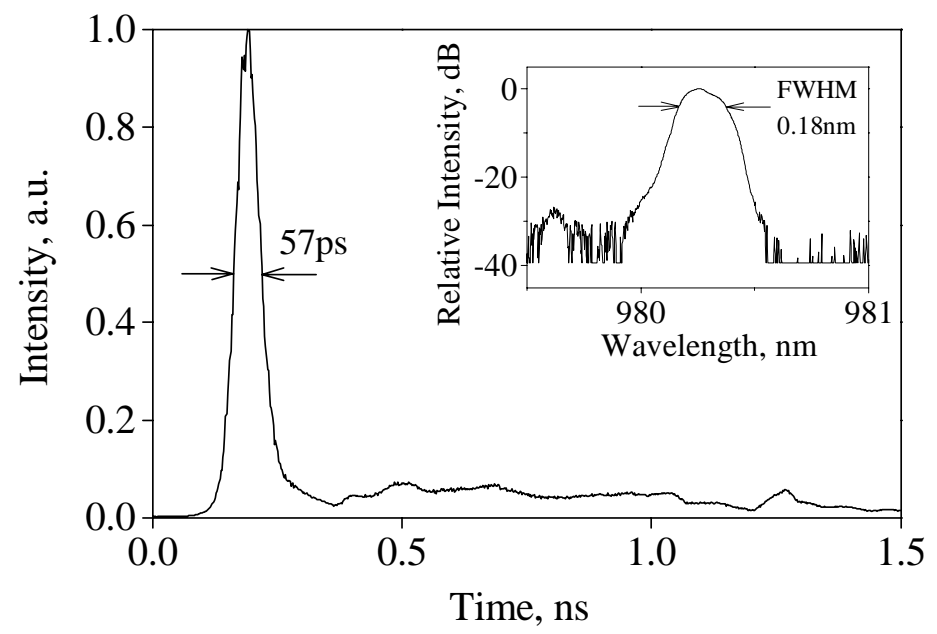

Fig.3: Tail-free gain-switched pulse $(\mathrm{FWHM}=57 \mathrm{ps})$ generated at maximum output power point. Inset shows the corresponding spectrum with FWHM bandwidth of $0.18 \mathrm{~nm}$ and side-band suppression ratio of $40 \mathrm{~dB}$.

The lateral intensity profile from the output facet measured in the near field with a CCD camera is presented in Fig.4. 
Filamentation in the laser beam was evident. However, the spectra and the temporal profiles of the emission in different filaments proved to be identical. The near-field mode pattern changed substantially with spectral tuning of the laser. We believe that lateral mode control can be achieved simultaneously with spectral control by using a specially designed external cavity.

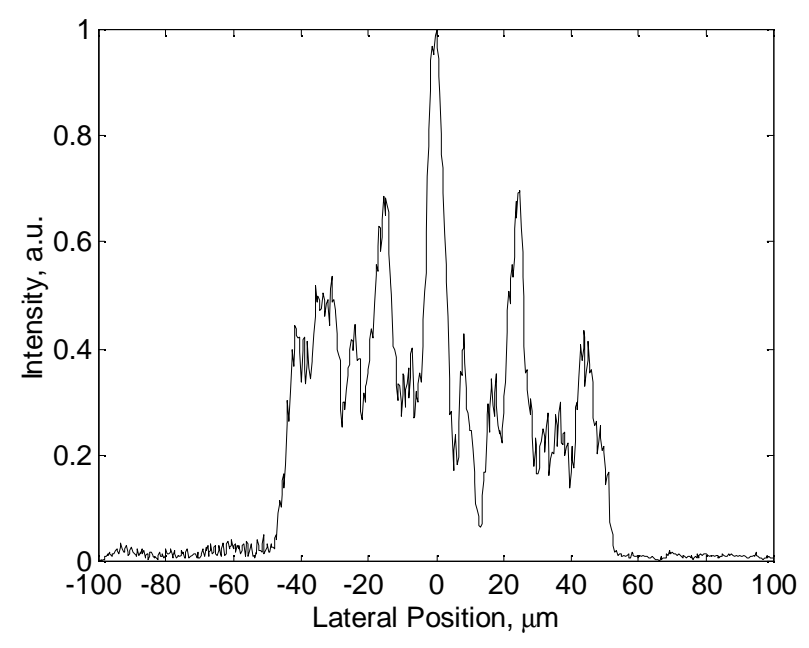

Fig.4: Lateral profile of the laser beam

\section{CONCLUSION}

In conclusion, generation of picosecond optical pulses with tunability over 100nm has been demonstrated for the first time using a grating coupled surface emitting laser in an optimised external cavity. Pulse duration in range of 40-100ps with the maximum peak power of $0.88 \mathrm{~W}$ and spectral side-band suppression ratio in excess of $40 \mathrm{~dB}$ has been achieved.

\section{REFERENCES}

1. J. Jiang, O. Smolski, C. Roychoudhuri, E. Portnoi, G. Venus, I. Gadjiev, J. McKillop, "Broad tunability of grating coupled surface-emitting laser with external cavity”, Electronics Letters, 35, pp. 1847-1848, 1999.

2. O. Smolski, J. Jiang, C. Roychoudhuri, E. Portnoi, J. Bullington, "Tunable picosecond pulses from gain-switched grating coupled surface emitting laser”, Novel In-Plane Semiconductor Lasers, paper No. 4651-09, Photonics West, SPIE, San Jose, California, USA, 2002. 